BULLETIN OF THE

AMERICAN MATHEMATICAL SOCIETY

Volume 80, Number 4, July 1974

\title{
EXTREMAL LENGTH, REPRODUCING DIFFERENTIALS AND ABEL'S THEOREM
}

\author{
BY CARL DAVID MINDA ${ }^{1}$
}

Communicated by F. W. Gehring, October 22, 1973

Let $c$ be a 1-chain on a Riemann surface $R$ and $\Gamma_{x}(R)$ a closed subspace of $\Gamma_{h}(R)$, the Hilbert space of square integrable harmonic differential forms on $R$, then there is a unique $\psi_{x}(c) \in \Gamma_{x}(R)$ such that $\int_{c} \omega=\left(\omega, \psi_{x}(c)\right)$ for all $\omega \in \Gamma_{x}(R) . \psi_{x}(c)$ is called the $\Gamma_{x}(R)$-reproducing differential for $c$ and $\left\|\psi_{x}(c)\right\|^{2}$ is a conformal invariant. For the case of a 1-cycle $c$ an extremal length interpretation for the squared norm of the reproducing differential was given by Accola [1] and Blatter [2] for $\Gamma_{h}(R)$, by Marden [3] for $\Gamma_{h o}(R)$ and by Rodin [5] for $\Gamma_{h s e}(R)$. In each of these results the curve family whose extremal length gave the square of the norm of the reproducing differential was a homology class associated with $c$. Rodin [5] asked whether there were similar theorems for other subspaces of $\Gamma_{h}(R)$ and what the proper curve family would be in case $c$ was an arbitrary 1-chain, not necessarily a 1-cycle. If $c$ is a single arc, then a reduced extremal distance interpretation of the norm of the reproducing differential for $\Gamma_{h e}(R), \Gamma_{h m}(R)$ and $\Gamma_{h e}(R) \cap \Gamma_{h s e}^{*}(R)$ was given in [4]. The purpose of this paper is to announce solutions to the problems posed by Rodin for a large number of important subspaces of $\Gamma_{h}(R)$; a complete, detailed paper is forthcoming.

For the sake of simplicity we shall consider only compact Riemann surfaces; this case gives rise to one of the most important applications. Let $c$ be a 1-chain on the compact Riemann surface $R$. Suppose that $\partial c=\sum_{j=1}^{J} n_{j} b_{j}-\sum_{i=1}^{I} m_{i} a_{i}$, where the points $a_{i}, b_{j}$ are all distinct and $m_{i}, n_{j}$ are positive integers, unless $\partial c=0$. Define $\mathscr{F}=\mathscr{F}(c)=\{d: d$ is a 1 -chain on $R$ and $\partial d=\partial c\}$ and $\mathscr{H}=\mathscr{H}(c)=\{d: d \in \mathscr{F}$ and $c-d$ is homologous to 0$\}$. Consider fixed local coordinates $w_{i}, z_{j}$ defined in a neighborhood of $a_{i}, b_{j}$ respectively. Given vectors $r=\left(r_{1}, \cdots, r_{I}\right)$ and $s=\left(s_{1}, \cdots, s_{J}\right)$ of positive numbers, let $R(r, s)$ be the bordered Riemann surface obtained by removing from $R$ disks of radius $r_{i}, s_{j}$ about $a_{i}, b_{j}$,

AMS (MOS) subject classifications (1970). Primary 30A52, 31 A15.

${ }^{1}$ Research supported in part by National Science Foundation Grant GP-39051. 
relative to these local coordinates. Set $\mathscr{F}(\boldsymbol{r}, s)=\{d \cap R(\boldsymbol{r}, \boldsymbol{s}): d \in \mathscr{F}\}$ and

$$
\tilde{\lambda}(\mathscr{F})=\lim _{r, s \downarrow 0} \lambda(\mathscr{F}(\boldsymbol{r}, s))+\frac{1}{2 \pi}\left(\sum_{i=1}^{I} m_{i}^{2} \log r_{i}+\sum_{j=1}^{J} n_{j}^{2} \log s_{j}\right) .
$$

$\tilde{\lambda}(\mathscr{F})$ exists and is called the reduced extremal length of the family $\mathscr{F}$ with respect to the local coordinates $w_{i}, z_{j}$. This quantity depends upon the choice of local coordinates in such a way that

$$
\exp (-2 \pi \tilde{\lambda}(\mathscr{F})) \prod\left|d w_{i}\right|^{m_{i}{ }^{2}} \prod\left|d z_{j}\right|^{n_{j}{ }^{2}}
$$

is an invariant form. $\tilde{\lambda}(\mathscr{H})$ is defined in a similar fashion.

We also associate two singular differentials with $c$. Let $p$ be a harmonic function on $R$ such that in a neighborhood of $a_{i}, p=\left(m_{i} / 2 \pi\right) \log \left|w_{i}-a_{i}\right|+$ $u_{i}$, where $u_{i}$ is harmonic at $a_{i}$, and near $b_{j}, p=-\left(n_{j} / 2 \pi\right) \log \left|z_{j}-b_{j}\right|+v_{j}$, where $v_{j}$ is harmonic at $b_{j}$. The function $p$ exists and is determined up to an additive constant. Set $\tilde{\psi}_{0}=\tilde{\psi}_{0}(c)=d p$ and $\tilde{\psi}_{h}=\tilde{\psi}_{h}(c)=\psi_{h}-\tilde{\psi}_{0}$, then for any $\omega \in \Gamma_{h}(R), 0=\left(\omega, \tilde{\psi}_{0}\right)$ and $\int_{c} \omega=\left(\omega, \tilde{\psi}_{h}\right)$. These inner products both exist since the integrals which give the inner products converge absolutely even though $\tilde{\psi}_{0}$ and $\tilde{\psi}_{h}$ have singularities. Set

$$
\left\langle\left\langle\tilde{\psi}_{h}\right\rangle\right\rangle^{2}=\lim _{r, s \downarrow 0}\left\|\tilde{\psi}_{h}\right\|_{R(r, s)}^{2}+\frac{1}{2 \pi}\left(\sum_{i=1}^{I} m_{i}^{2} \log r_{i}+\sum_{j=1}^{J} n_{j}^{2} \log s_{j}\right) .
$$

This quantity exists but is not invariantly defined; however,

$$
\exp \left(-2 \pi\left\langle\left\langle\tilde{\psi}_{h}\right\rangle\right\rangle^{2}\right) \prod\left|d w_{i}\right|^{m_{i}{ }^{2}} \prod\left|d z_{j}\right|^{n_{j}{ }^{2}}
$$

is an invariant form. $\left\langle\left\langle\tilde{\psi}_{0}\right\rangle\right\rangle^{2}$ is defined analogously. It can be shown that $\left\langle\left\langle\tilde{\psi}_{h}\right\rangle\right\rangle^{2}-\left\langle\left\langle\tilde{\psi}_{0}\right\rangle\right\rangle^{2}=\left\|\psi_{h}\right\|^{2}$.

The following theorem is our main result.

THEOREM. $\quad \tilde{\lambda}(\mathscr{F}(c))=\left\langle\left\langle\tilde{\psi}_{0}(c)\right\rangle\right\rangle^{2}$ and $\tilde{\lambda}(\mathscr{H}(c))=\left\langle\left\langle\tilde{\psi}_{h}(c)\right\rangle\right\rangle^{2}$.

Corollary. $\left\|\psi_{h}(c)\right\|^{2}=\tilde{\lambda}(\mathscr{H}(c))-\tilde{\lambda}(\mathscr{F}(c))$.

This corollary leads to an extremal length interpretation of Abel's theorem. Let $D$ be a divisor on the compact Riemann surface $R$. Assume that either $D=0$ or $D=B-A$, where $A$ and $B$ are disjoint integral divisors; that is, $A=\sum_{i=1}^{I} m_{i} a_{i}$ and $B=\sum_{j=1}^{J} n_{j} b_{j}$, the points $a_{i}, b_{j}$ all being distinct and $m_{i}, n_{j}$ being positive integers. $D$ is called a principal divisor if there is a rational function $f$ on $R$ such that the divisor of $f$ is $D$. Abel's theorem asserts that $D$ is a principal divisor if and only if there is a 1-chain $c$ on $R$ with the property that $\partial c=D$ and $\int_{c} \omega=0$ for all $\omega \in \Gamma_{h}(R)$. Now, in order that $\int_{c} \omega=0$ holds for all $\omega \in \Gamma_{h}(R)$, it is necessary and sufficient that $\left\|\psi_{h}(c)\right\|=0$. Consequently, the next theorem has been established. 
THEOREM. A divisor $D$ on a compact Riemann surface $R$ is principal if and only if there is $a 1$-chain $c$ on $R$ with $\partial c=D$ and $\tilde{\lambda}(\mathscr{F}(c))=\tilde{\lambda}(\mathscr{H}(c))$.

Our main theorem has several analogs on an open Riemann surface. In fact, on an open surface there are six curve families associated with a 1-chain $c$. The reduced extremal length of all six families can be expressed in terms of singular differentials which are closely related to various reproducing differentials connected with $c$. By making use of these results, we can give an extremal length interpretation for the squared norm of the $\Gamma_{x}(R) \cap \Gamma_{y}^{*}(R)$-reproducing differential for a 1-chain $c$; here $x$ and $y$ can represent any one of $h, h s e, h o, h e, h m$, except that $x=y=h e$ or ho is not permitted. There are fourteen nontrivial such subspaces of $\Gamma_{h}(R)$.

\section{REFERENCES}

1. R. D. M. Accola, Differentials and extremal length on Riemann surfaces, Proc. Nat. Acad. Sci. U.S.A. 46 (1960), 540-543. MR 22 \#9598.

2. C. Blatter, Über Extremallängen auf geschlossenen Flächen, Comment. Math. Helv. 35 (1961), 153-168. MR 24 \#A1389.

3. A. Marden, The weakly reproducing differentials on open Riemann surfaces, Ann. Acad. Sci. Fenn. Ser. A I No. 359 (1965), 32 pp. MR 32 \#5868.

4. C. D. Minda, Extremal length and harmonic functions on Riemann surfaces, Trans. Amer. Math. Soc. 171 (1972), 1-22.

5. B. Rodin, Extremal length of weak homology classes on Riemann surfaces, Proc. Amer. Math. Soc. 15 (1964), 369-372. MR 29 \#240.

Department of Mathematics, University of Cincinnati, Cincinnati, Ohio 45221 